\title{
Multi-point Adjoint-Based Design of Tilt-Rotors in a Noninertial Reference Frame
}

\author{
William T. Jones, ${ }^{*}$ Eric J. Nielsen, ${ }^{\dagger}$ and Elizabeth M. Lee-Rausch ${ }^{\ddagger}$ \\ NASA Langley Research Center, Hampton, VA 23681-2199 \\ Cecil W. Acree, Jr. ${ }^{\S}$ \\ NASA Ames Research Center, Moffett Field, CA 94035-1000
}

\begin{abstract}
Optimization of tilt-rotor systems requires the consideration of performance at multiple design points. In the current study, an adjoint-based optimization of a tilt-rotor blade is considered. The optimization seeks to simultaneously maximize the rotorcraft figure of merit in hover and the propulsive efficiency in airplane-mode for a tilt-rotor system. The design is subject to minimum thrust constraints imposed at each design point. The rotor flowfields at each design point are cast as steady-state problems in a noninertial reference frame. Geometric design variables used in the study to control blade shape include: thickness, camber, twist, and taper represented by as many as 123 separate design variables. Performance weighting of each operational mode is considered in the formulation of the composite objective function, and a build up of increasing geometric degrees of freedom is used to isolate the impact of selected design variables. In all cases considered, the resulting designs successfully increase both the hover figure of merit and the airplane-mode propulsive efficiency for a rotor designed with classical techniques.
\end{abstract}

\section{Nomenclature}

$F M \quad$ Rotorcraft figure of merit

$\mathbf{F}_{v} \quad$ Viscous flux tensor

I Identity tensor

K Elasticity coefficient matrix

$k \quad$ Thermal conductivity

$N \quad$ Number of blades

$\hat{\mathbf{n}} \quad$ Outward-pointing unit normal vector

$P \quad$ Pressure

$p \quad$ User-defined exponent

Q Vector of conserved variables

R Spatial residual vector

r Position vector

$R \quad$ Blade radius

$r \quad$ Radial station

S Source term vector

$\begin{array}{ll}S & \text { Control volume surface area } \\ T & \text { Temperature } \\ t & \text { Time } \\ U & \text { Velocity } \\ u, v, w & \text { Cartesian components of velocity } \\ u_{i} & \text { Cartesian directional displacements } \\ V & \text { Volume of control volume } \\ \mathbf{X} & \text { Vector of mesh coordinates } \\ x_{i} & \text { Cartesian coordinate directions } \\ & \\ \text { Subscripts } \\ \text { cruise } & \text { Cruise condition } \\ e & \text { Effective } \\ f & \text { Flow field } \\ h o v e r & \text { Hover condition } \\ i, j, k & \text { Indices } \\ m & \text { Mesh } \\ m p & \text { Multi-point composite } \\ Q & \text { Torque } \\ \text { surf } & \text { Surface quantity } \\ T & \text { Thrust }\end{array}$

\footnotetext{
*Computer Engineer, Computational AeroSciences Branch, MS 128, AIAA Associate Fellow

${ }^{\dagger}$ Research Scientist, Computational AeroSciences Branch, MS 128, Senior Member AIAA

$\ddagger$ Research Engineer, Computational AeroSciences Branch, MS 128, Senior Member AIAA

$\S$ Aerospace Engineer, Aeromechanics Branch, MS 243-12, Senior Member AIAA
} 


$\begin{array}{ll}\text { tip } & \text { Rotor tip } \\ \text { Symbols } \\ \mathcal{O}() & \text { Order of Magnitude } \\ \alpha & \text { User-defined weighting factor } \\ \Delta & \text { Difference } \\ \varepsilon & \text { Strain tensor } \\ \eta & \text { Propulsive efficiency } \\ \Theta & \text { Blade collective pitch setting } \\ \boldsymbol{\Lambda} & \text { Adjoint variable vector } \\ \lambda, \mu & \text { Linear elasticity Lamé constants }\end{array}$

$\begin{array}{ll}\nu & \text { Poisson's ratio } \\ \rho & \text { Density } \\ \sigma & \text { Rotor solidity } \\ \tau & \text { Viscous stress tensor } \\ \Omega & \text { Angular velocity vector } \\ \omega & \text { User-defined weighting factor } \\ \infty & \text { Freestream quantity } \\ \text { Superscripts } \\ T & \text { Vector transpose } \\ * & \text { User-defined target }\end{array}$

\section{Introduction}

APLication of high-fidelity computational fluid dynamics (CFD) has become commonplace in the fixedAwing aerospace community. Software packages that solve the Euler equations and Reynolds-averaged Navier-Stokes equations on both structured and unstructured meshes are now used routinely by aerodynamicists in the analysis and design of new configurations. Moreover, as algorithms and computer hardware have continued to mature, the coupling of formal design optimization techniques and sufficiently accurate physical models has become a viable solution for the design of many large scale aerospace configurations.

The application of high-fidelity CFD tools to the analysis and design of full rotorcraft configurations is considerably challenging. Such flowfields are inherently unsteady, frequently involve fluid velocities that range from quiescent to transonic flow, and typically require the simulation of complex aerodynamic and aerostructural interactions between dynamic vehicle components. Recent literature suggests that the use of high-fidelity CFD methods in this regime is growing, but the computational cost that is required to capture the necessary spatial and temporal scales of a typical rotorcraft flowfield remains considerable. ${ }^{1-9}$

In the field of gradient-based design, adjoint methods are known to provide an extremely efficient means for computing sensitivity information. The cost of such methods is equivalent to the expense that is associated with solving the analysis problem and is independent of the number of design variables. Adjoint methods can also be used to perform mathematically rigorous mesh adaptation and error estimation. Significant success has been reported for the application of these techniques to steady problems. ${ }^{10-14}$

In general, optimization and mesh adaptation for large-scale unsteady flows based on adjoint methods require a time-dependent implementation of the equations. Considerable effort by a number of research groups is being focused in this area, and examples of the use of such approaches have recently emerged. ${ }^{15-17}$ Despite the algorithmic efficiency, however, the computational cost of these general time-dependent approaches can be considerable.

An adjoint-based design capability was previously demonstrated for rotor configurations for which the analysis problem may be cast as a steady problem in a noninertial reference frame. ${ }^{18}$ This approach permitted the use of an existing steady-state adjoint formulation with minor modifications to perform sensitivity analyses. The resulting formulation was valid for isolated rigid rotors in hover or where the freestream velocity is aligned with the axis of rotation.

The goal of the current work is to apply the non-inertial capability ${ }^{18}$ to the multi-point design of a tilt-rotor blade. The design will simultaneously optimize aerodynamic performance of the rotor at both hover and cruise conditions. For hover, the objective is to maximize the rotor figure of merit while maintaining a minimum thrust coefficient. For cruise, the objective is to maximize the cruise performance efficiency while maintaining a minimum thrust coefficient. To better understand the contributions of the chosen geometric design variables, the design follows an orderly build up of increasing geometric degrees of freedom.

\section{Flow Equations}

The governing equations for the flowfield are the compressible, perfect gas Reynolds-averaged Navier-Stokes equations written in a reference frame that is rotating with a constant angular velocity, $\boldsymbol{\Omega}$ :

$$
\frac{\partial(\mathbf{Q} V)}{\partial t}+\oint_{\partial V}\left(\mathbf{F}_{i}-\mathbf{F}_{v}\right) \cdot \hat{\mathbf{n}} d S=\mathbf{S}
$$


where $\mathbf{Q}$ is the vector of volume-averaged conserved variables, $\mathbf{Q}=[\rho, \rho u, \rho v, \rho w, E]^{T}, \hat{\mathbf{n}}$ is an outward-pointing unit normal, and $V$ is the control volume bounded by the surface $\partial V$. The inviscid and viscous flux tensors are given by

$$
\mathbf{F}_{i}=\left[\begin{array}{c}
\rho(\mathbf{u}-\boldsymbol{\Omega} \times \mathbf{r}) \\
\rho \mathbf{u}^{T}(\mathbf{u}-\boldsymbol{\Omega} \times \mathbf{r})+P \mathbf{I} \\
(\mathbf{u}-\boldsymbol{\Omega} \times \mathbf{r})(E+P)+(\boldsymbol{\Omega} \times \mathbf{r}) P
\end{array}\right]
$$

and

$$
\mathbf{F}_{v}=\left[\begin{array}{c}
0 \\
\boldsymbol{\tau} \\
\mathbf{u} \cdot \boldsymbol{\tau}-k \nabla T
\end{array}\right]
$$

The source term $\mathbf{S}$ represents a Coriolis effect that results from the rotating frame of reference:

$$
\mathbf{S}=\left[\begin{array}{c}
0 \\
-\rho(\boldsymbol{\Omega} \times \mathbf{u}) \\
0
\end{array}\right]
$$

Here, $\mathbf{u}$ is the absolute velocity vector, $\mathbf{u}=[u, v, w]^{T}, \mathbf{r}$ is the position vector relative to the axis of rotation, and $\tau$ is the viscous stress tensor. The equations are closed with the perfect gas equation of state and an appropriate turbulence model for the eddy viscosity. For rotorcraft simulations, the formulation that is described here is applicable to rigid tilt-rotor geometries in either a hover, ascending/descending, or airplane mode cruise flight condition, where the freestream velocity vector is parallel to the angular velocity vector, $\boldsymbol{\Omega}$.

The FUN3D flow solver that is used in the current work ${ }^{19-22}$ can be used to perform aerodynamic simulations across the speed range, and an extensive list of options and solution mechanisms is available for spatial and temporal discretizations on general static or dynamic mixed-element unstructured meshes that may or may not contain overset mesh topologies.

In the current study, the spatial discretization uses a finite-volume approach in which the dependent variables are stored at the vertices of single-block tetrahedral meshes. Inviscid fluxes at cell interfaces are computed using the upwind scheme of Roe, ${ }^{23}$ and viscous fluxes are formed using an approach that is equivalent to a central difference Galerkin procedure. The eddy viscosity is modeled using the one-equation approach of Spalart and Allmaras ${ }^{24}$ with the source term modification proposed by Dacles-Mariani et al. ${ }^{25}$ For the steady-state flows (relative to the noninertial reference frame) that are described in this study, temporal discretization is performed using a backward-Euler scheme with local time stepping. Scalable parallelization is achieved through domain decomposition and message-passing communication.

An approximate solution of the linear system of equations that is formed within each time step is obtained through several iterations of a multicolor Gauss-Seidel point-iterative scheme. The turbulence model is integrated all the way to the wall without the use of wall functions. The turbulence model is solved separately from the mean flow equations at each time step with a time integration and a linear system solution scheme that is identical to that employed for the mean flow equations.

\section{Mesh Equations}

To deform the interior of the computational mesh as the surface mesh evolves during a shape-optimization procedure, the mesh is assumed to obey the linear elasticity equations of solid mechanics. These relations can be written as

$$
\oint_{\partial V} \lambda\left(\sum_{i=1}^{3} \frac{\partial u_{i}}{\partial x_{i}}\right) \mathbf{I} \cdot \hat{\mathbf{n}} d S+\oint_{\partial V} 2 \mu \varepsilon \cdot \hat{\mathbf{n}} d S=0
$$


where

$$
\varepsilon=\frac{1}{2}\left(\frac{\partial u_{i}}{\partial x_{j}}+\frac{\partial u_{j}}{\partial x_{i}}\right)
$$

is the strain tensor, $u_{i}$ is the displacement vector in each of the Cartesian coordinate directions, $x_{i}$, and $\lambda$ and $\mu$ are material properties of the elastic medium. The quantities $\lambda$ and $\mu$ are related to Young's modulus, E, and Poisson's ratio, $\nu$, through the following:

$$
\lambda=\frac{\nu E}{(1+\nu)(1-2 \nu)}
$$

and

$$
\mu=\frac{E}{2(1+\nu)}
$$

The system is closed with the specification of two of the four parameters $\lambda, \mu, E$, and $\nu$. In the current implementation, $E$ is taken as inversely proportional to the distance from the nearest solid boundary, while Poisson's ratio is taken uniformly as zero. This approach forces all cells that are near boundaries to move in a nearly rigid fashion, while cells that are far from the boundaries are allowed to deform more freely. The system of equations is solved using GMRES $^{26}$ with either a point-implicit or ILU(0) preconditioning technique. ${ }^{22,27}$

\section{Discrete Adjoint Equations}

To derive the discrete adjoint equations, a compact notation is introduced for the governing equations that are outlined above. The spatial residual vector $\mathbf{R}$ of Eq. (1) is defined as

$$
\mathbf{R} \equiv \oint_{\partial V}\left(\mathbf{F}_{i}-\mathbf{F}_{v}\right) \cdot \hat{\mathbf{n}} d S-\mathbf{S}
$$

Furthermore, the linear system of equations given by Eq. (5) can be written as

$$
\mathbf{K X}=\mathbf{X}_{\text {surf }}
$$

where $\mathbf{K}$ is the elasticity coefficient matrix that results from the discretization of Eq. (5), $\mathbf{X}$ is the vector of the mesh-point coordinates, and $\mathbf{X}_{\text {surf }}$ is the vector of known surface mesh-point coordinates, complemented by zeros for all interior coordinates. With the approach that was taken by Nielsen, ${ }^{11}$ a Lagrangian function can be defined as follows:

$$
L\left(\mathbf{D}, \mathbf{Q}, \mathbf{X}, \boldsymbol{\Lambda}_{f}, \boldsymbol{\Lambda}_{m}\right)=f(\mathbf{D}, \mathbf{Q}, \mathbf{X})+\mathbf{\Lambda}_{f}^{T} \mathbf{R}(\mathbf{D}, \mathbf{Q}, \mathbf{X})+\mathbf{\Lambda}_{m}^{T}\left(\mathbf{K X}-\mathbf{X}_{s u r f}\right)
$$

where $\mathbf{D}$ represents a vector of design variables, $f$ is an objective function, and $\boldsymbol{\Lambda}_{f}$ and $\boldsymbol{\Lambda}_{m}$ are the adjoint variables that multiply the residuals of the flow and the mesh equations. In this manner, the governing equations may be viewed as constraints.

Differentiating Eq. (11) with respect to $\mathbf{D}$ and equating the $\partial \mathbf{Q} / \partial \mathbf{D}$ and $\partial \mathbf{X} / \partial \mathbf{D}$ coefficients to zero yields the discrete adjoint equations for the flowfield and mesh, respectively:

$$
\left[\frac{\partial \mathbf{R}}{\partial \mathbf{Q}}\right]^{T} \boldsymbol{\Lambda}_{f}=-\frac{\partial f}{\partial \mathbf{Q}}
$$


and

$$
\mathbf{K}^{T} \boldsymbol{\Lambda}_{m}=-\left\{\frac{\partial f}{\partial \mathbf{X}}+\left[\frac{\partial \mathbf{R}}{\partial \mathbf{X}}\right]^{T} \boldsymbol{\Lambda}_{f}\right\}
$$

The remainder of the terms in the linearized Lagrangian can be grouped to form an expression for the final sensitivity vector:

$$
\frac{d L}{d \mathbf{D}}=\frac{\partial f}{\partial \mathbf{D}}+\boldsymbol{\Lambda}_{f}^{T} \frac{\partial \mathbf{R}}{\partial \mathbf{D}}-\boldsymbol{\Lambda}_{m}^{T}\left[\frac{\partial \mathbf{X}_{\text {surf }}}{\partial \mathbf{D}}\right]
$$

Equations (12) and (13) provide an efficient means for determining discretely consistent sensitivity information. The expense that is associated with solving these equations is independent of $\mathbf{D}$ and is similar to that of the governing equations. After the solutions for $\boldsymbol{\Lambda}_{f}$ and $\boldsymbol{\Lambda}_{m}$ have been determined, then the desired sensitivities may be calculated using Eq. (14), for which the computational cost is negligible.

A discrete adjoint implementation has been developed ${ }^{11,17,21,27,28}$ for the flow solution method that is described above. The flowfield adjoint equations are solved in an exact dual manner, which guarantees an asymptotic convergence rate that is identical to the primal problem and costate variables that are discretely adjoint at every iteration of the solution process. The grid adjoint equations are solved using GMRES in a manner that is identical to the method used for Eq. (5). To accommodate the noninertial reference frame used in the current study, minor modifications have been made to include the effects of the mesh speeds and the Coriolis terms.

\section{Design Methodology}

\section{A. Design Variables}

The implementation that is described by Nielsen ${ }^{11}$ is sufficiently general such that the user is able to employ a geometric parameterization scheme of choice, provided that the associated linearizations required by the adjoint method described above are also available. For the current study, a mesh parameterization scheme tailored for aerodynamic shape optimization is used. ${ }^{29}$ This approach can be used to define very general shape parameterizations of existing grids using a set of aircraft-centric design variables, such as camber, thickness, shear, twist, and planform parameters at various locations on the geometry. The user also has the freedom to directly associate two or more design variables to create more general parameters. In the current work, this option is used to link several piecewise twist variables across the span of a rotor blade to create a single twist variable that is used to prescribe the blade collective pitch setting, $\Theta$. The option is used similarly to link planform variables to control blade taper. In the event that multiple bodies of the same shape are to be designed, as in the case of rotor geometries, the implementation allows a single set of design variables to be used to simultaneously define all bodies as a group. In this manner, all bodies in the group have the same shape at a given design cycle throughout the course of the design.

\section{B. Objective and Constraint Functions}

The implementation of Nielsen ${ }^{11}$ permits multiple objective functions, $f_{i}$, and explicit constraints, $c_{j}$, of the following form, each containing a summation of $n_{i}$ and $m_{j}$ individual components, respectively:

$$
f_{i}=\sum_{k=1}^{n_{i}} \omega_{k}\left(C_{k}-C_{k}^{*}\right)^{p_{k}}
$$

and

$$
c_{j}=\sum_{k=1}^{m_{j}} \omega_{k}\left(C_{k}-C_{k}^{*}\right)^{p_{k}}
$$


Here, $\omega_{k}$ represents a user-defined weighting factor, $C_{k}$ is an aerodynamic coefficient such as total drag or the pressure or viscous contributions to such quantities, and $p_{k}$ is a user-defined exponent. The $*$ superscript indicates a user-defined target value of $C_{k}$. Furthermore, the user may specify the boundaries in the grid to which each component function applies.

\section{Design Points and Optimization Strategies}

The current implementation supports an arbitrary number of user-specified design points at which objective and constraint functions may be posed. Each design point may be defined by a variation of basic flowfield quantities such as the Mach number, or a more general characteristic such as a specific computational grid that is appropriate for each individual design point. In the current study, the two design points use their own distinct baseline mesh, one for hover and the other for the cruise condition. While it would have been possible to elastically deform a single mesh to accommodate the changes in $\Theta$ for the two conditions, the individual meshes were generated to insure the highest quality baselines about which subsequent design driven shape changes would be incorporated through elastic deformation.

To perform multi-point optimization, a composite objective function, $f_{m p}$, can be defined based on the individual objective functions, $f_{i}$, posed at each design point. In this work, the composite objective function, $f_{m p}$, defines a linear combination of the objective functions for the hover and cruise conditions:

$$
f_{m p}=\alpha_{\text {hover }} f_{\text {hover }}+\alpha_{\text {cruise }} f_{\text {cruise }}
$$

where $\alpha$ is a constant weighting factor for each point that is applied to the corresponding objective at the point.

The multi-point approach that is used here is commonly used to combine point solutions to multi-objective optimization problems into a single scalar objective. The difficulty is that out of the range of many possible solutions only one is obtained by setting some parameters heuristically and externally, for example the weights of the composite scalar objective. The investigation of more sophisticated optimization strategies is out of scope of the current work. In principle the simple strategy adopted here is sufficient for the problem of interest and the investigation of more sophisticated optimization strategies is relegated to future work.

SNOPT $^{30,31}$ is used to solve the optimization problem defined above subject to explicit constraints specified at each design point. The optimization algorithm is allowed to perform a maximum of 20 design cycles with a maximum of 30 function evaluations per cycle, and considers the design converged and exits if it believes the current objective function matches the value at the optimal solution to four significant digits. Constraints are considered satisfied if their values do not exceed the specified bounds by $0.1 \%$ of the bound value. The design at the initial choice of $\mathrm{D}$ is not required to satisfy the constraints; if needed, the optimizer attempts to locate a feasible starting point on its own.

\section{Design Case}

The subject of the current study is the three-bladed Tilt Rotor Aeroacoustics Model (TRAM) ${ }^{32,33}$ shown in Figure 1. The TRAM represents an optimized system designed with traditional tools. It was selected for this study because the model readily exists and is an adequate surrogate for the more sensitive V-22. The original quarter scale TRAM model was resized to match full scale flight conditions. The multi-point optimization considers both hover and airplane mode cruise conditions similar to those found in open literature for the V-22. In hover, the tip Mach number is 0.707 , the freestream temperature is $519^{\circ}$ Rankine, and the Reynolds number is 9.2 million, based on the blade tip chord of 22 inches. The cruise condition was modeled at an altitude of 3,000 feet, a tip Mach number of 0.650 , a freestream temperature of $551.5^{\circ}$ Rankine, and a Reynolds number of 6.1 million (again based on the blade tip chord).

A separate baseline mesh was generated for each of the two conditions of the design study. The mesh for the hover evaluation contained 8,105,037 nodes and 47,884,674 tetrahedral elements and was designed for a nominal collective pitch setting $\Theta$ of $14^{\circ}$. The cruise condition mesh contained 8,106,096 nodes and 47,891,211 tetrahedral elements at a nominal $\Theta$ of $41^{\circ}$. The same relative spacing constraints were used to generate both meshes as indicated by the similar sizes of the meshes. The surface grid for one of the blades is shown in Figure 1(b). All of the grids have been generated with VGRID. ${ }^{34}$ The blade trailing edges are blunt. 


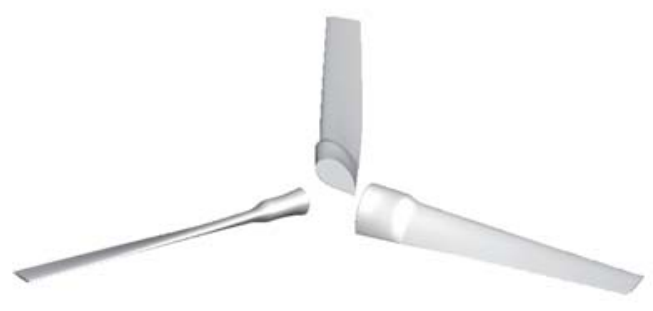

(a) Rotor surface geometry

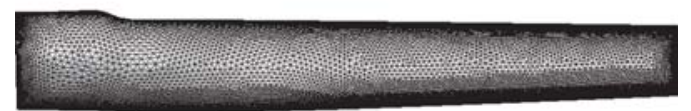

(b) Typical blade surface mesh used for design computations

Figure 1. Tilt Rotor Aeroacoustics Model (TRAM)

A geometric parameterization ${ }^{29}$ has been developed for the baseline blade geometry, as shown in Figure 2. The approach yields a total of 123 active design variables, including 50 variables to control the blade thickness, 60 variables to control the blade camber, 10 to control the blade twist, and 1 to control the blade taper. The final two variables were defined to address the collective pitch angles, $\Theta$, for each design point. These were formed by linking together all of the blade twist variables at a given design point into a single design variable. A separate design variable was required for each design point as control of $\Theta$ must be independent at each point. The taper design variable was also formed by linking together the two in-plane components of the planform variables at the blade tip. However, in this linking, the components were linked such that the movement of the aft planform variable was three times the magnitude of the forward variable and in the opposite direction. This was defined such that the $\frac{1}{4}$-chord line would remain invariant to changes in taper. To further facilitate the invariance, the $\frac{1}{4}$-chord line of the blade was aligned to the parameterization reference frame through a series of rigid body transformations prior to parameterization.

Bounds on the design variables have been initially chosen with the intent to prevent nonphysical surface shapes; only thickness changes that increased the section thickness were allowed to eliminate thinning of the rotor blades. The parameterization also allows for radial blade shearing deformations, however these variables are not active design variables in the current study. All blades at a given design point are grouped together such that a perturbation of the design variables produces the same blade shape for the rotor system. Other than the collective pitch angle, $\Theta$, a common parameterization is used between design points. This maintains the same blade shape across the design.

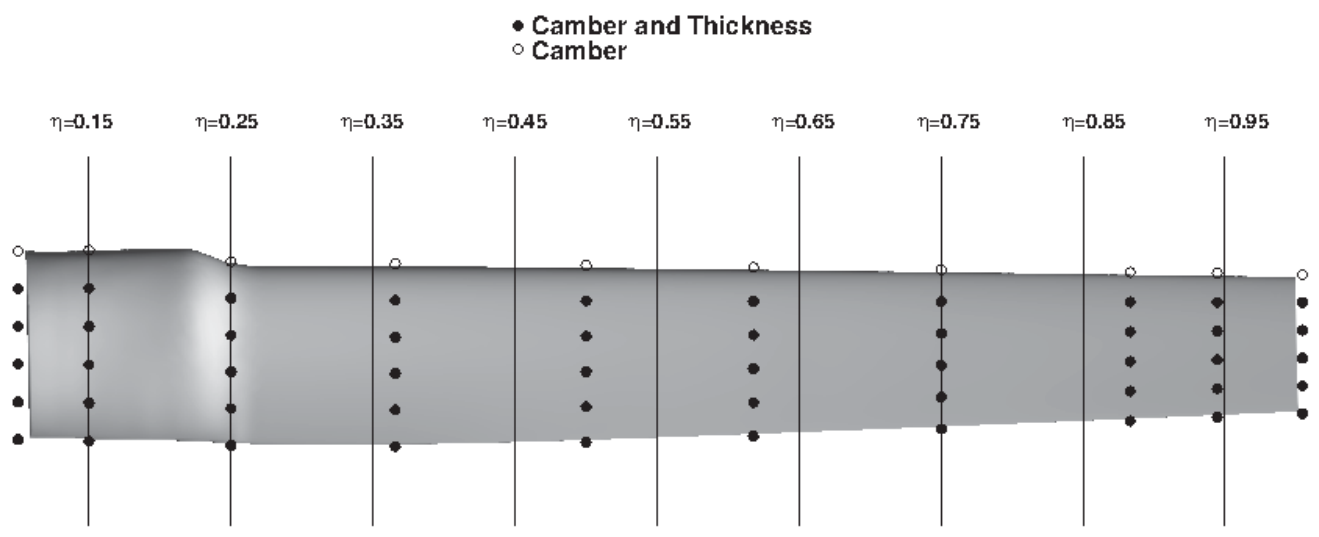

Figure 2. Design variable and radial blade locations.

The design problem definition calls for explicit constraints on the thrust coefficient, $C_{T}$, at each condition. Prior to the multi-point design, a simple preliminary design optimization was performed for each condition 
to determine the $\Theta$ required to trim the rotor to the target $C_{T}$. The trimming was performed as two separate single-point unconstrained optimizations where $\Theta$ was the only design variable and the respective $C_{T}$ represented a single objective for each point. The resulting changes to $\Theta$ were modest, as will be shown below. Again, it is not required that the constraints be satisfied to begin the optimization, however, this did demonstrate the ability of the optimizer to trim the rotor to a target thrust.

For the hover condition, a single objective is used, where $\omega=1, p=2$, and $C$ is defined as the square of the commonly used rotorcraft figure of merit, $F M$, which is composed of the rotor thrust, $C_{T}$, and torque, $C_{Q}$, coefficients:

$$
C=F M^{2}=\frac{C_{T}^{3}}{2 C_{Q}^{2}}
$$

The square has been introduced to avoid the appearance of a square root of a negative thrust value in the linearized form of the objective function. The value of $C^{*}$, of Eq. (15), is chosen to be 2, which is considerably larger than both the baseline value and the theoretical maximum value of 1 . By defining $C^{*}$ and $p$ in this way, minimization of the objective will minimize the difference between the actual and target coefficients in Eq. (15) effectively maximizing the value of $F M$. The explicit thrust constraint was used to set a lower bound on $C_{T}$ of 0.0158 with $\omega$ chosen so that the constraint was of $\mathcal{O}(1)$.

The cruise condition also used a single objective function with $\omega=1, p=2$, and $C$ defined as the airplane mode propulsive efficiency $\eta$ :

$$
C=\eta=\frac{C_{T} U_{\infty}}{C_{Q} U_{t i p}}
$$

Again, the value of $C^{*}$ is chosen to be 2, which is considerably larger than both the baseline value and the theoretical maximum value of $\eta=1$. Minimization of the objective will again maximize $\eta$. The explicit thrust constraint placed a lower bound on $C_{T}$ of 0.0027 where $\omega$ was again chosen so that the constraint was of $\mathcal{O}(1)$.

All computations were performed using the NASA Advanced Supercomputing (NAS) high performance computing resources. A design cycle requires at a minimum a single function and gradient evaluation for the current value of $\mathbf{D}$. A function evaluation in this context consists of an evaluation of the surface parameterization for each blade, a solution of Eq. (5) to deform the interior of the mesh according to the current surface grid, and a solution of the flow equations, Eq. (1). Using the adjoint approach that is outlined above, a gradient evaluation requires a solution of the flowfield adjoint equations, Eq. (12); a solution of the mesh adjoint equations, Eq. (13); an evaluation of the linearized surface parameterization for each blade; and, finally, an evaluation of the gradient expression given by Eq. (14). The convergence criteria that are used for each of the solvers has a direct impact on the efficiency. Note, the time that is required to solve Eqs. (1) and (12) tends to decrease toward the end of an optimization as the design converges and the solution restarts become more effective.

\section{E. Design Considerations}

In tilt-rotor design, rotor efficiency must often be a trade off between airframe and wing efficiency while aerodynamic efficiency is traded against weight and mission efficiency (e.g. total fuel burn). This leads to a paradox: maximizing aerodynamic efficiency of an isolated rotor does not guarantee maximum operating efficiency, and may even lead to a poorly designed aircraft.

In hover, tilt-rotor thrust must match weight plus download. In cruise, thrust must match drag, including induced drag from the airframe and wing. Consider a cruise velocity determined by mission requirements and a wing design optimized for that velocity. If the mission is fixed and the aircraft is not resized, then neither hover nor cruise thrust should change. Any improvement provided by aerodynamic optimization should be manifest as a reduction in power (equivalently represented ${ }^{35}$ by $C_{Q}$ in Eqs. (18) and (19)), not an increase in thrust or airspeed.

Furthermore, while in hover most of the rotor power results from induced power. However, in cruise the power is primarily dependent on profile power, which varies little with thrust when a well designed rotor is operating at its design condition. Therefore, in high-speed cruise, $C_{T}$ can be increased with negligible increase 
in profile power, resulting in a large increase in $\eta$. However, the increased thrust cannot be effectively utilized by an aircraft operating at fixed velocity given a fixed value of airframe drag. Alternatively, increasing the velocity at fixed power may require a heavier wing to satisfy aeroelastic stability margins. As a result, increases in drag or weight that result from higher cruise $C_{T}$ may result in worse overall mission performance than a configuration with lower $C_{T}$ and lower $\eta$. Hence the above paradox that maximizing rotor efficiency may not maximize operating efficiency.

The argument may be inverted when considering the trade-offs between cruise and hover efficiency. The aircraft may benefit from a smaller rotor with higher cruise $C_{T}$, which would give a lower $F M$ but higher $\eta$. Such a result should not be assumed in advance, however. The optimum design may require a larger rotor with higher $F M$ and lower $\eta$. In either case, rotor performance trends for both hover and cruise must be considered based on a consistent reference rotor size, which is determined by the thrust needed for hover.

The above discussion is predicated on the assumption that one sizes the aircraft to some combination of hover and cruise efficiency. But there are other design constraints not mentioned, notably engine-out and maneuver requirements. Design for engine-out conditions is implicitly included in a design to maximum $F M$. Design for engine-out requires the maximum possible thrust at a strict power limit, which also maximizes $F M$ by definition. Design for maneuvers, however, generally requires maximization of available thrust, which will not occur at maximum FM. Worse, critical maneuvers, particularly low-speed turns, do not have axial flow at the rotor, even for tilt-rotors or tilt-wings. In turning flight, the total lift required is greater than the weight. For tilt-rotors, the worst-case turning condition will occur at low speed with the rotor in edgewise flight or tilted slightly forward, and with the wing carrying only part of the total lift.

As such, aerodynamic optimization based on thrust, as distinct from maximum efficiency, is nevertheless required. If greater thrust can be achieved at a given rotor size, then the aircraft can be resized with a smaller rotor. A smaller rotor will have both lower profile power and higher $C_{T}$ in cruise, which would automatically provide higher $\eta$ without compromising airframe aerodynamics. A sizing code can be used to determine the proper trade-off between maximizing thrust in maneuvers and maximizing $F M$ in hover with aerodynamic optimization via CFD utilizing a consistent rotor size. For acceptable CPU time, and to minimize problems with convergence, an axial-flow surrogate for a turning condition can be used whereby a constraint is added that requires some minimum value of thrust at nominal operating conditions, where the specified $C_{T}$ is greater than that at which $F M$ is maximized.

Thrust can be increased by increasing blade chord, but this does not increase $F M$ and decreases $\eta$ because profile drag is higher. To properly separate the effects of blade area from the effects of optimized airfoils, twist, and planform, rotor performance coefficients should be scaled by the ratio of blade area to disk area, or solidity, $\sigma$. Local blade section velocity, dynamic pressure and Mach number all increase with radius, so designing rotors to thrust-weighted solidity ${ }^{36}$ is common:

$$
\sigma=\frac{N c_{e}}{\pi R}
$$

where

$$
c_{e}=\frac{\int_{0}^{R} c r^{2} d r}{\int_{0}^{R} r^{2} d r}
$$

At the time of this writing, the full linearization of the blade loading $\left(C_{T} / \sigma\right)$, namely the linearization of $\sigma$, was not available. Therefore, it was not possible to use blade loading as an explicit constraint in this design. As an alternative, we imposed a minimum constraint on $C_{T}$ as mentioned above for each mode of flight.

\section{F. Design Breakdown}

To better understand the contributions of airfoil shape, twist, and planform toward optimized rotor design, the design was broken into four distinct steps referenced A-D. Step A sought to optimize airfoil camber and thickness distributions at radial increments along the blade to maximize hover $F M$ and cruise $\eta$ while keeping airfoil twist and planform fixed. A hover $F M$ to cruise performance $\eta$ weighting of 2:1 was selected by setting the weights of the composite objective function of Eq. (17) as $\alpha_{\text {hover }}=2.0$ and $\alpha_{\text {cruise }}=1.0$. Step $\mathrm{B}$ added airfoil twist as an additional design variable to Step A while maintaining the same 2:1 hover-to-cruise performance weighting. Step C built upon previous steps to add blade taper. Again the 2:1 ratio of $F M$ to $\eta$ was maintained. Finally, Step D repeated Step C with the hover-to-cruise weighting reversed from 2:1 to 1:2, $\alpha_{\text {hover }}=1.0$ and $\alpha_{\text {cruise }}=2.0$. The design steps are summarized in Table 1 . 
Table 1. Breakdown of design steps

\begin{tabular}{ccccccc}
\hline \hline Step & Camber & Thickness & Twist & Taper & $\alpha_{\text {hover }}$ & $\alpha_{\text {cruise }}$ \\
\hline A & $\checkmark$ & $\checkmark$ & & & 2.0 & 1.0 \\
B & $\checkmark$ & $\checkmark$ & $\checkmark$ & & 2.0 & 1.0 \\
C & $\checkmark$ & $\checkmark$ & $\checkmark$ & $\checkmark$ & 2.0 & 1.0 \\
D & $\checkmark$ & $\checkmark$ & $\checkmark$ & $\checkmark$ & 1.0 & 2.0 \\
\hline
\end{tabular}

\section{Results}

The multi-point design objective was to maximize both the $F M$ in hover and $\eta$ in cruise. The optimization was simultaneously required to maintain or exceed the baseline thrust coefficients for each operational mode. In addition to the shape variables active for each design step, a decoupled collective pitch design variable was defined at each design point resulting in two additional design variables per step.

All optimizations were executed on 2,048 Intel ${ }^{\circledR}$ Sandybridge processing cores of the NAS Pleiades system. Note that the wall time is not a linear function of number of function evaluations due to many complicating factors (each function evaluation may require a different number of iterations to converge, a design cycle may require multiple function/gradient evaluations, etc.). Step A activated 110 shape variables to control airfoil camber and thickness. With the addition of a $\Theta$ variable for each of the two design points, the optimization of 112 variables required 17 function evaluations and roughly 20 hours of wall time. The addition of blade twist in step B resulted in 120 shape variables. A total of 48 function evaluations were required to optimize the 122 variables, and roughly 49 hours of wall time.

Both Steps C and D employed all 121 active shape variables to optimize airfoil camber, thickness, twist, and blade taper. Addition of the decoupled $\Theta$ design variables defined for each design point resulted in 123 design variables in total. In these remaining steps, the composite design objectives were subject to two inversely proportional performance weightings. Step C required 29 function evaluations and roughly 53 hours of wall time. Step D required 51 function evaluations and roughly 43 hours of wall time.

\section{A. Hover Design Point}

Tables 2 and 3 detail the output from each step in the design buildup for the hover point. For each step in the tables, the optimized collective pitch angle, $\Theta$, is given along with the values of the thrust constraint, $C_{T}$, blade loading $C_{T} / \sigma$, and figure of merit, $F M$. Also shown is the difference in the figure of merit, $\Delta F M$, and its percent change from the baseline value, \%Change.

Table 2. Figure of Merit function (Constant $C_{T}$ )

\begin{tabular}{ccccccc}
\hline \hline Step & $\Theta_{\text {hover }}$ & $C_{T}$ & $\frac{C_{T}}{\sigma}$ & FM & $\Delta$ FM & \%Change \\
\hline Baseline & $13.91^{\circ}$ & 0.0158 & 0.1505 & 0.7564 & - & - \\
$\mathrm{A}$ & $13.52^{\circ}$ & 0.0159 & 0.1514 & 0.7685 & 0.0121 & 1.600 \\
$\mathrm{~B}$ & $14.60^{\circ}$ & 0.0158 & 0.1505 & 0.7640 & 0.0076 & 1.005 \\
$\mathrm{C}$ & $13.83^{\circ}$ & 0.0158 & 0.1365 & 0.7707 & 0.0143 & 1.891 \\
$\mathrm{D}$ & $14.22^{\circ}$ & 0.0158 & 0.1386 & 0.7603 & 0.0039 & 0.516 \\
\hline
\end{tabular}

Table 3. Figure of Merit function ( $C_{T}$ increase allowed)

\begin{tabular}{ccccccc}
\hline \hline Step & $\Theta_{\text {hover }}$ & $C_{T}$ & $\frac{C_{T}}{\sigma}$ & FM & $\Delta$ FM & \%Change \\
\hline Baseline & $13.91^{\circ}$ & 0.0158 & 0.1505 & 0.7564 & - & - \\
A & $13.47^{\circ}$ & 0.0169 & 0.1610 & 0.7714 & 0.0150 & 1.983 \\
B & $11.61^{\circ}$ & 0.0160 & 0.1524 & 0.7828 & 0.0264 & 3.490 \\
$\mathrm{C}$ & $12.85^{\circ}$ & 0.0175 & 0.1563 & 0.7840 & 0.0276 & 3.649 \\
$\mathrm{D}$ & $12.17^{\circ}$ & 0.0168 & 0.1556 & 0.7819 & 0.0255 & 3.371 \\
\hline
\end{tabular}

Table 2 relates the cases where an equality constraint was imposed to hold $C_{T}$ at or near its baseline value. 
Table 3 shows data from the optimization where $C_{T}$ was allowed to vary above the baseline value. Recall Section V, E explained why arbitrary increases in $C_{T}$ may not result in an good design. However, in the absence of a blade loading constraint, the data of Table 3 show that increases in $C_{T}$, such as would accompany maintaining $C_{T} / \sigma$ for steps $\mathrm{C}$ and $\mathrm{D}$, result in further increases in $F M$.

Also note that the trimmed baseline value of $\Theta$ is quite close to the nominal value of $\Theta=14^{\circ}$ used to develop the mesh. Each design step showed a modest increase in the $F M$ with the greatest increase shown for Step C. Considering Table 3, it is expected that an equality constraint on $C_{T} / \sigma$ holding to the baseline value of 0.1505 would produce better than a $2.0 \%$ increase in $F M$.

\section{B. Cruise Design Point}

Tables 4 and 5 represent the results for the design buildup at the airplane-mode cruise point. Here the optimized collective pitch angle, $\Theta$, is given along with the values of the thrust constraint, $C_{T}$, blade loading, $C_{T} / \sigma$, and propulsive efficiency, $\eta$. Also shown is the difference in the propulsive efficiency, $\Delta \eta$, and its percent change from the baseline value, \%Change.

Similar to the hover data, Table 4 represents the cases where an equality constraint was imposed to hold $C_{T}$ at or near its baseline value of 0.0027 . Table 5 shows data from the optimization where $C_{T}$ was allowed to vary above the baseline value. The impact of increased $C_{T}$ on $\eta$ is clearly shown in Table 5 . While not all aircraft can exploit the increased $\eta$ shown in Table 5 , the results illustrate the potential gains in cruise efficiency where the aircraft design space allows increases in $C_{T}$.

Table 4. Propulsive efficiency (Constant $C_{T}$ )

\begin{tabular}{ccccccc}
\hline \hline Step & $\Theta_{\text {cruise }}$ & $C_{T}$ & $\frac{C_{T}}{\sigma}$ & $\eta$ & $\Delta \eta$ & \%Change \\
\hline Baseline & $41.37^{\circ}$ & 0.0027 & 0.0257 & 0.6738 & - & - \\
$\mathrm{A}$ & $40.98^{\circ}$ & 0.0028 & 0.0267 & 0.6816 & 0.0078 & 1.158 \\
$\mathrm{~B}$ & $41.78^{\circ}$ & 0.0027 & 0.0257 & 0.6971 & 0.0233 & 3.458 \\
$\mathrm{C}$ & $41.71^{\circ}$ & 0.0027 & 0.0233 & 0.7023 & 0.0285 & 4.230 \\
$\mathrm{D}$ & $42.05^{\circ}$ & 0.0027 & 0.0234 & 0.7008 & 0.0270 & 4.007 \\
\hline
\end{tabular}

Table 5. Propulsive efficiency ( $C_{T}$ increase allowed)

\begin{tabular}{ccccccc}
\hline \hline Step & $\Theta_{\text {cruise }}$ & $C_{T}$ & $\frac{C_{T}}{\sigma}$ & $\eta$ & $\Delta \eta$ & \%Change \\
\hline Baseline & $41.37^{\circ}$ & 0.0027 & 0.0257 & 0.6738 & - & - \\
$\mathrm{A}^{1}$ & $42.50^{\circ}$ & 0.0076 & 0.0724 & 0.8243 & 0.1505 & 22.34 \\
$\mathrm{~B}^{1}$ & $42.50^{\circ}$ & 0.0098 & 0.0933 & 0.8491 & 0.1753 & 26.02 \\
$\mathrm{C}^{1}$ & $42.50^{\circ}$ & 0.0089 & 0.0795 & 0.8469 & 0.1731 & 25.69 \\
$\mathrm{D}^{1}$ & $42.50^{\circ}$ & 0.0098 & 0.0907 & 0.8506 & 0.1768 & 26.24 \\
\hline
\end{tabular}

${ }^{1} \Theta$ at Upper bound

Here too, the trimmed baseline value of $\Theta$ is near the nominal $\Theta=41^{\circ}$ used to construct the reference mesh. Each design step showed an increase in the propulsive efficiency with the greatest increases shown for Steps $\mathrm{C}$ and $\mathrm{D}$ where the planform was increased. It is reasonable to assume from this data that increases in $\eta$ in excess of $4 \%$ for Steps $\mathrm{C}$ and $\mathrm{D}$ can be expected as $C_{T} / \sigma$ is allowed to approach the baseline value of $0.0257 \%$.

Note that for each design step when $C_{T}$ was constrained to be greater than the baseline value, the collective pitch angle $\Theta$ was increased to its upper bound for the design of $42.5^{\circ}$. This accounts for much of the increase in $C_{T}$ and further points to the need for a true $C_{T} / \sigma$.

\section{Design Results}

Both the hover and cruise design points show modest increases in both the $F M$ and $\eta$ with increasing geometric degrees of freedom. The weighting used in Step C increased the hover FM a modest $1.89 \%$ over the baseline value for fixed $C_{T}$, while the cruise performance increased $4.23 \%$ over the baseline. When inverting the 
weighting for Step D to 1:2, the hover figure of merit improvement dropped to $0.52 \%$ while the cruise $\eta$ maintained improvement at $4.0 \%$.

Figures 3-6 represent airfoil sections from $r / R=0.20$ to $r / R=1.0$ for each of the design Steps A-D respectively. Note that perturbations to the blade collective pitch $\Theta$ have been removed to allow comparison to the baseline shapes that are represented by the solid black lines. Also note that the vertical scale has been exaggerated to emphasize the shape differences. The airfoil shapes representing the design configuration are represented with dotted red lines in each figure.

In Figure 3, modest shape changes are observed for Step A with the most notable changes occurring outboard of the $r / R=0.50$ section. Increases in camber are observed near the trailing edge for sections $r / R=0.50$ through 0.95. Thickness changes are most notable for sections $r / R=0.60$ to 0.95 . Figure 4 shows the same airfoil sections for Step B. The shape changes resulting from the addition of the blade twist variable are more readily apparent and are now observed over the entire blade span from $r / R=0.20$ to 1.0 . In addition, the rotor blade appears to exhibit some washout near the tip from $r / R=0.90$ to 1.0 .

The optimized airfoil sections resulting from Step C are shown in Figure 5. The addition of blade taper as a design variable is reflected in the increased chord of the outboard sections. While this change in chord does alter rotor solidity, the reader is reminded that no constraint on rotor solidity has been imposed in the current study. However, the 1/4-chord location is invariant to changes in chord resulting from altering blade taper. Notice also the upsweep to the trailing edge of the rotor tip at $r / R=1.0$.

Step D shape results are recorded in Figure 6. Again, the modifications are observed over the entire blade radius. Chord changes through the taper variable have been allowed without regard to changes in rotor solidity.

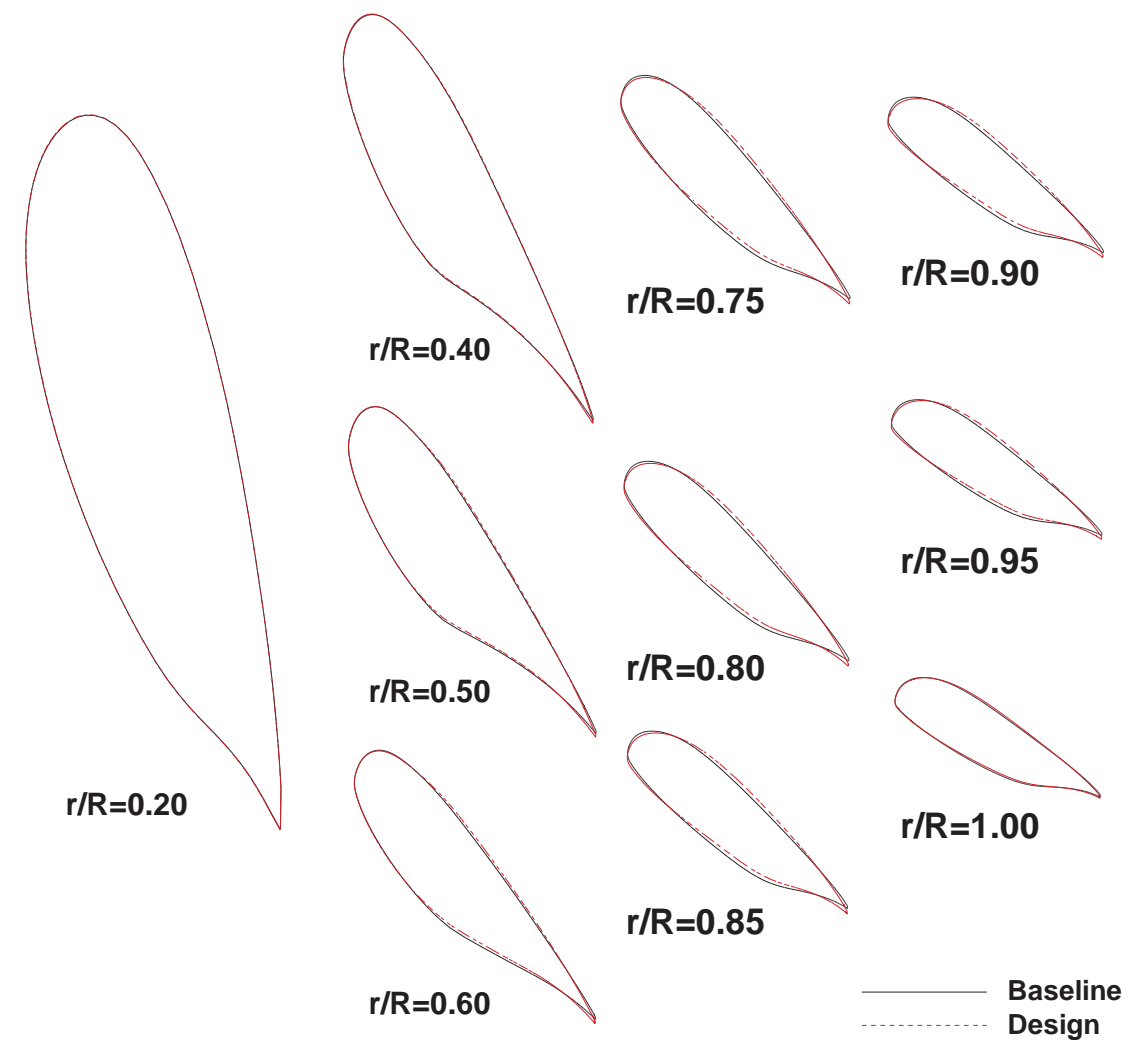

Figure 3. Step A: Composite Weight 2:1, Optimize Thickness, Camber, Collective. Collective perturbation removed and vertical scale exaggerated for comparison. 


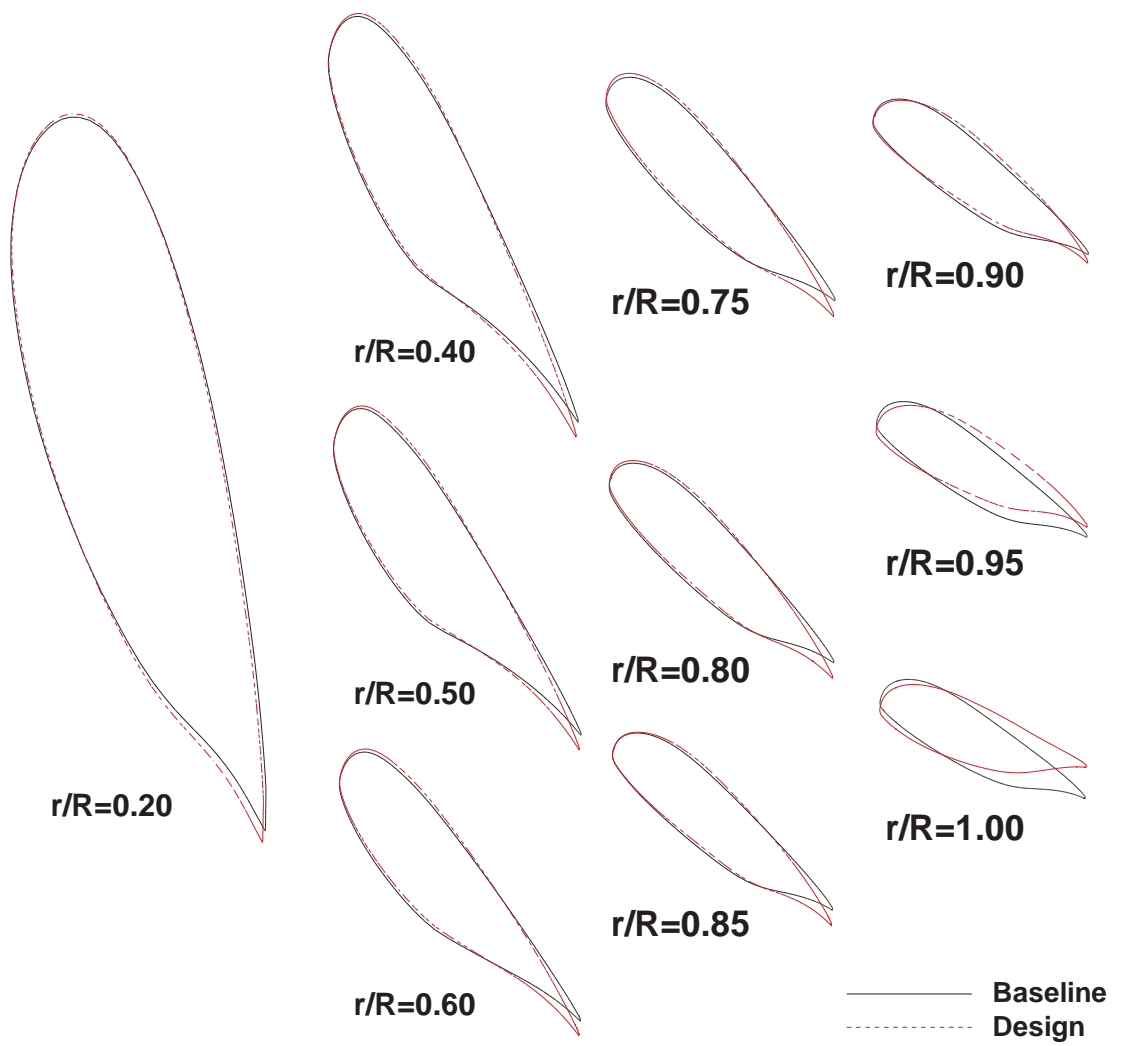

Figure 4. Step B: Composite Weight 2:1, Optimize Thickness, Camber, Twist, Collective. Collective perturbation removed and vertical scale exaggerated for comparison.

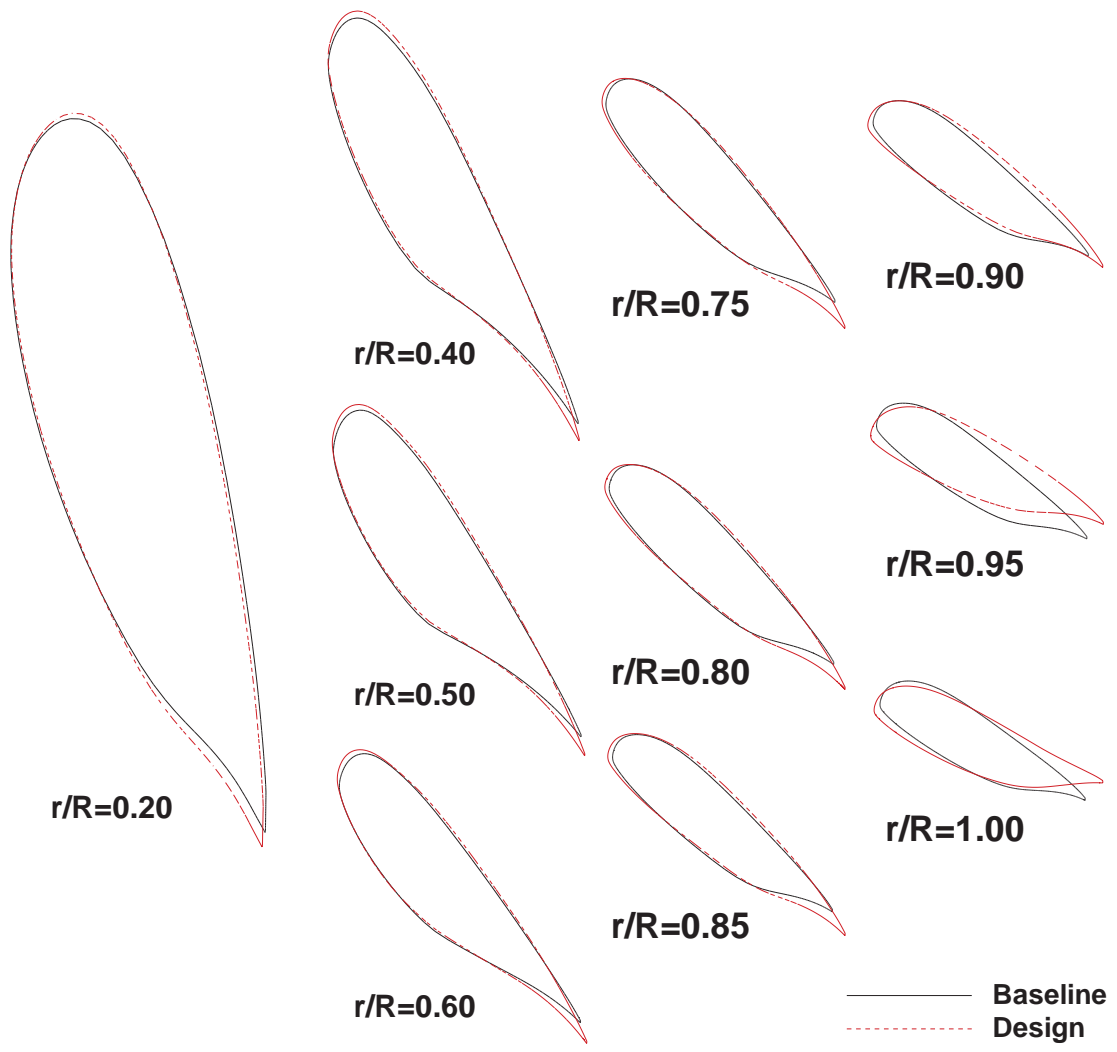

Figure 5. Step C: Composite Weight 2:1, Optimize Thickness, Camber, Twist, Taper, Collective. Collective perturbation removed and vertical scale exaggerated for comparison. 


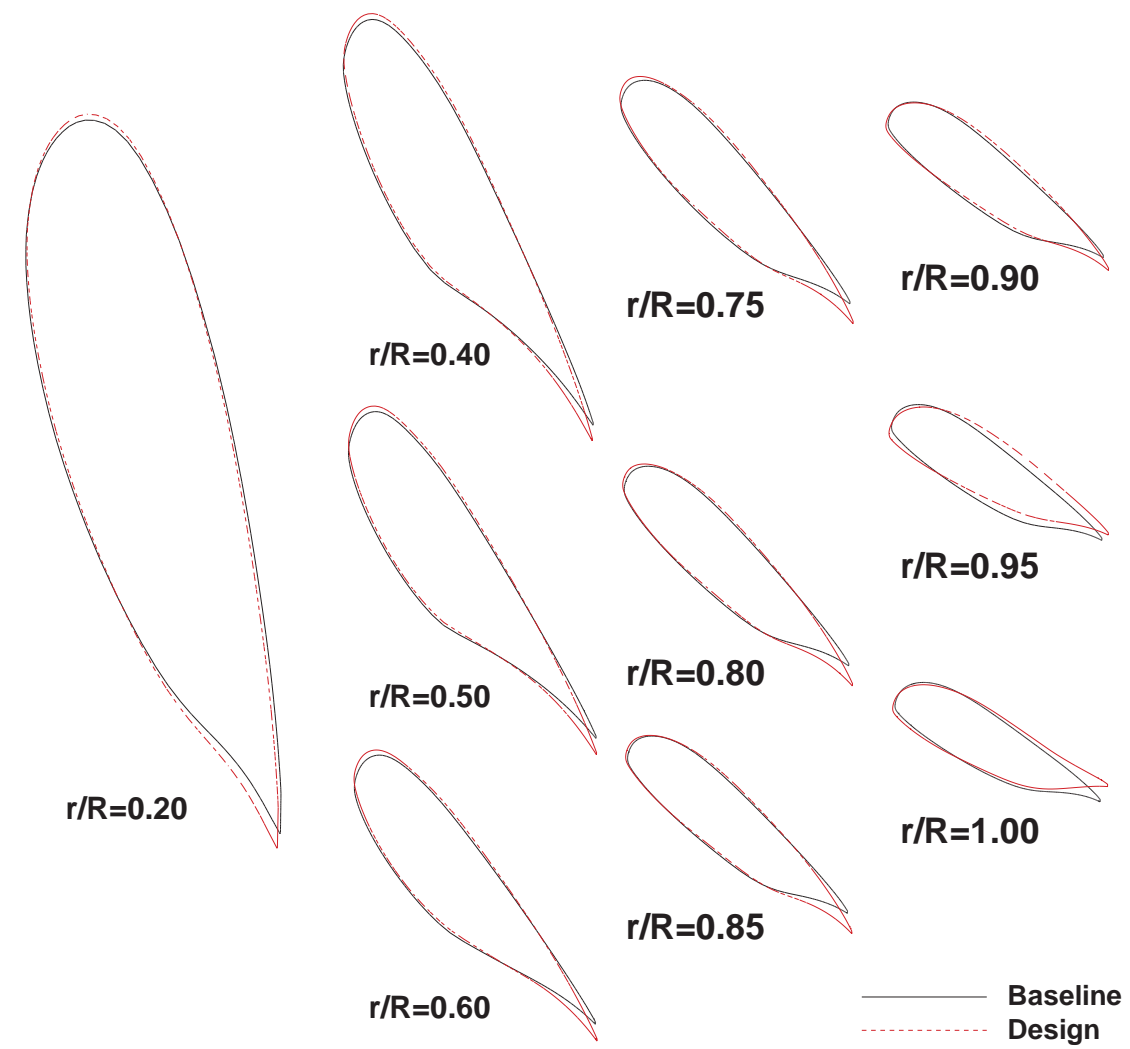

Figure 6. Step D: Composite Weight 1:2, Optimize Thickness, Camber, Twist, Taper, Collective. Collective perturbation removed and vertical scale exaggerated for comparison.

The design history for Steps A-D are represented in Figures 7-10 where the thrust constraint, $C_{T}$, was held above the baseline value. Here $C_{T}$, the objective for each design point $F M$ and $\eta$, and the composite objective function are plotted against design cycle. In each plot, the thrust constraint minimum values are also shown for reference. For each design step, both objectives increase rapidly during the first few design cycles. Recall that the optimization problem is formulated as a minimization of the composite objective function hence it exhibits inverse behavior as it decreases with design cycle.

\section{Conclusion}

A discrete adjoint-based methodology for performing design optimization of isolated rotor problems that appear as steady flows in a noninertial reference frame has been used to drive a multi-point optimization of a tilt-rotor configuration. A composite multi-point objective function was constructed using performance weighting between the two design points and the optimization was subject to constraints on the thrust at each design point. Data was presented for optimization considering a constant thrust and a minimum thrust constraint. The optimization successfully increased the hover figure of merit and the airplane-mode propulsive efficiency in all cases considered. To better understand the impact of the chosen design variables, a four step approach was taken to incrementally increase the geometric degrees of freedom in the design. The results illustrated the need to impose a blade loading constraint to limit increases in cruise $C_{T}$ when designing to fixed mission requirements. Nevertheless, the optimization showed further improvement for the TRAM rotor previously designed with traditional techniques.

\section{Acknowledgments}

This work was performed under the Rotary Wing project of the NASA Fundamental Aeronautics Program. The authors would like to thank Robert Biedron for the development of many of the rotorcraft capabilities in FUN3D and Gloria Yamauchi of the Ames Aeromechanics Branch for help in developing the design problem. 


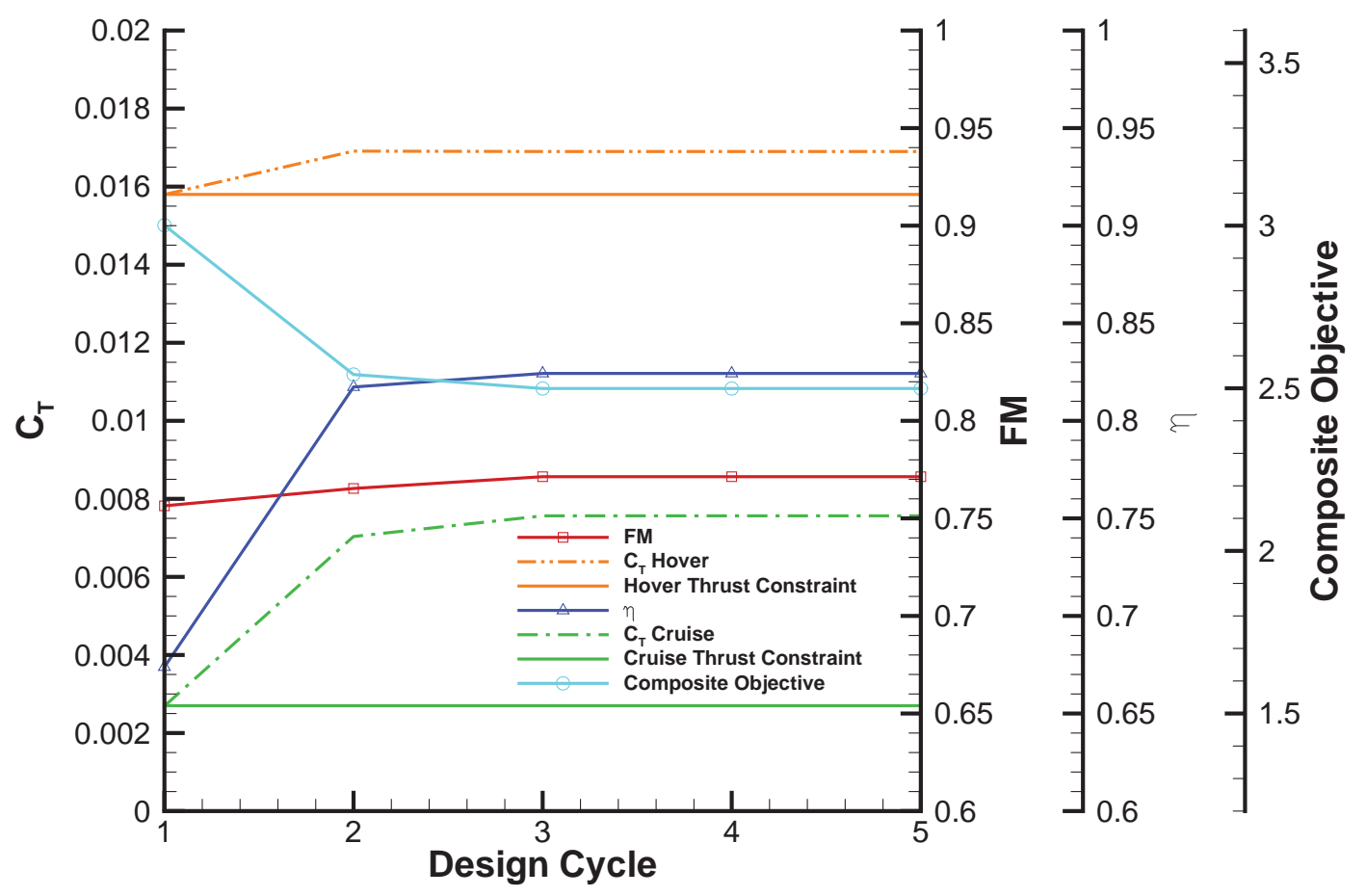

Figure 7. Step A: Composite Weight 2:1, Optimize Thickness, Camber, Collective.

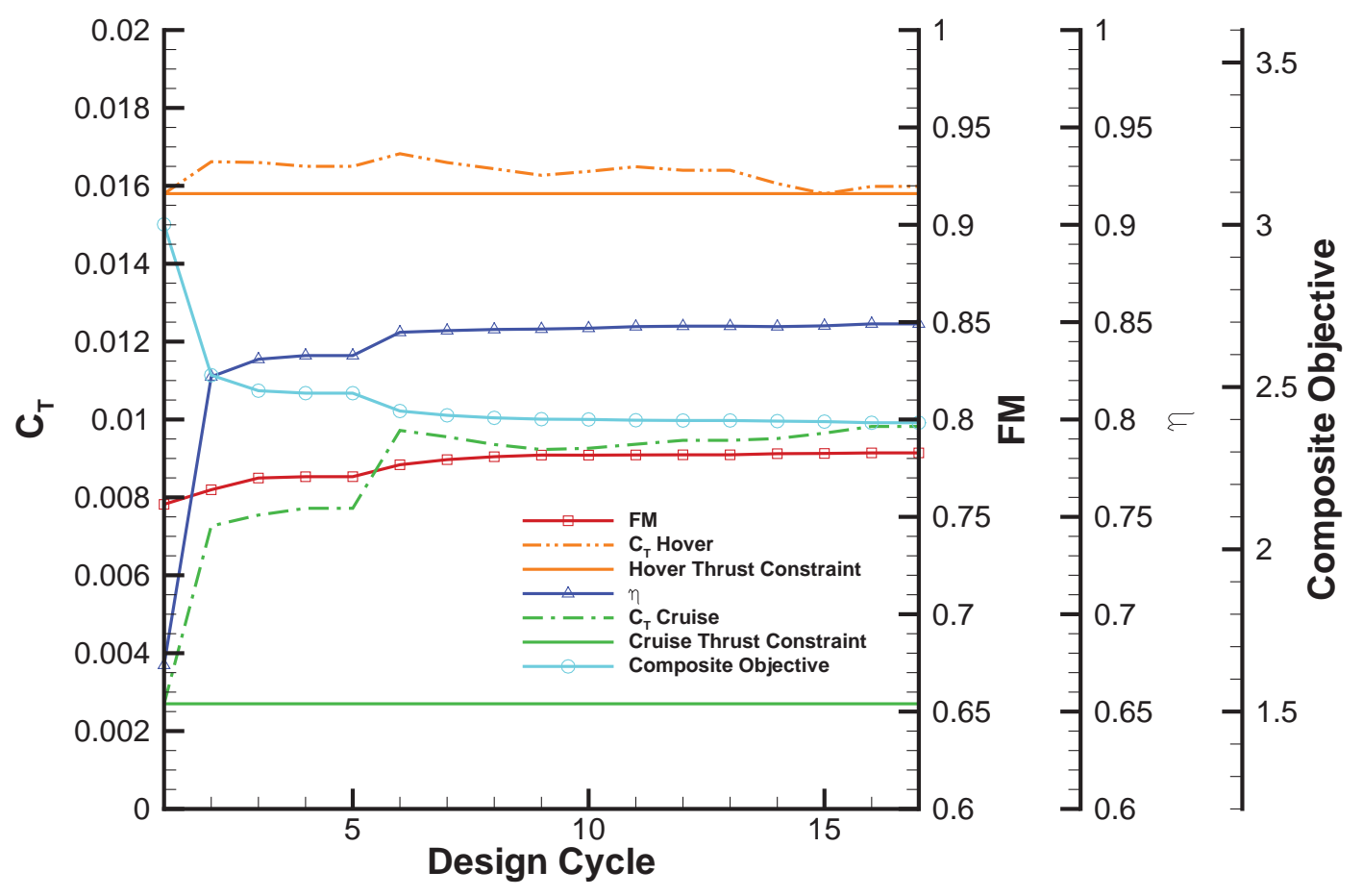

Figure 8. Step B: Composite Weight 2:1, Optimize Thickness, Camber, Twist, Collective. 


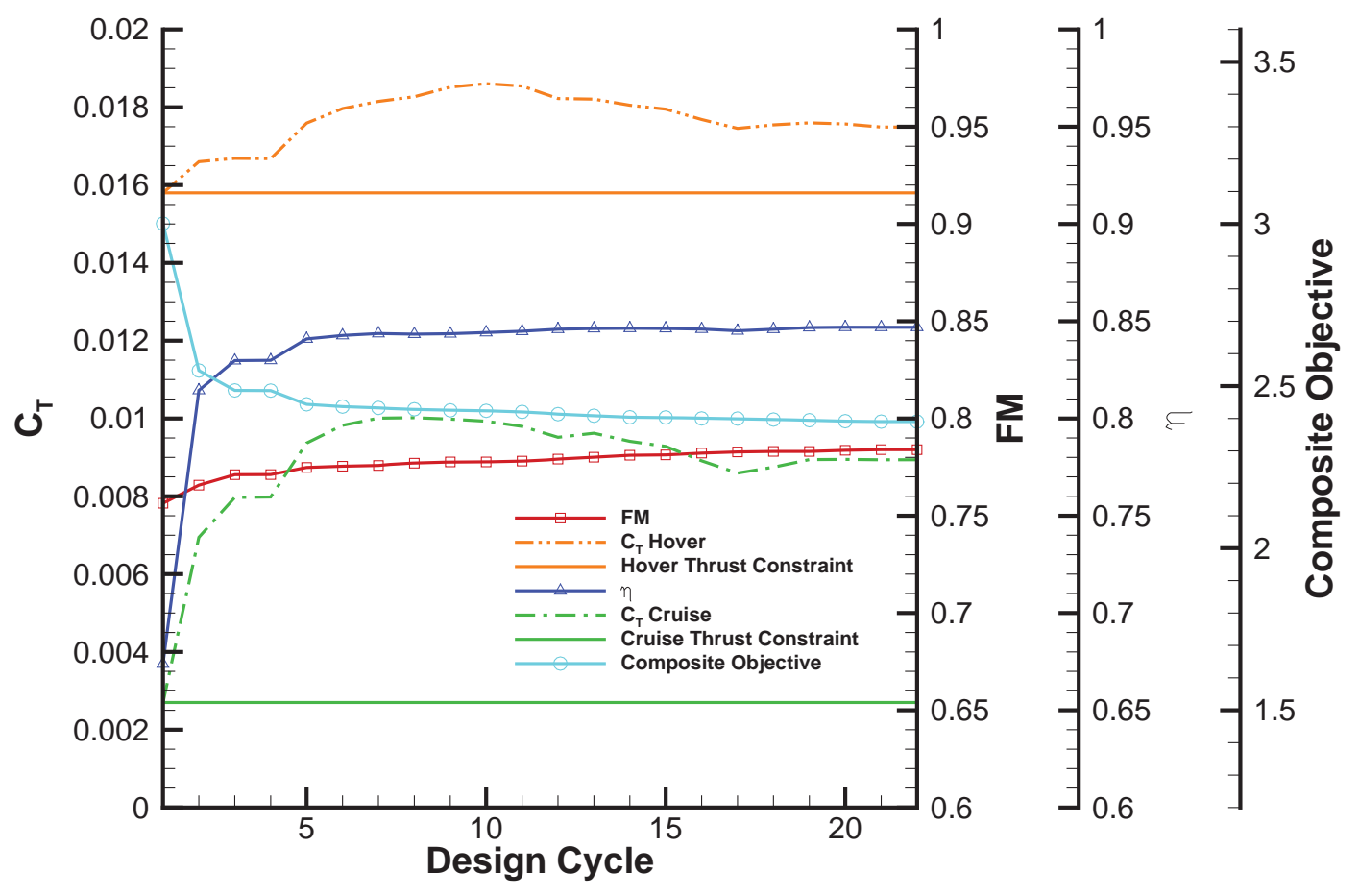

Figure 9. Step C: Composite Weight 2:1, Optimize Thickness, Camber, Twist, Taper, Collective.

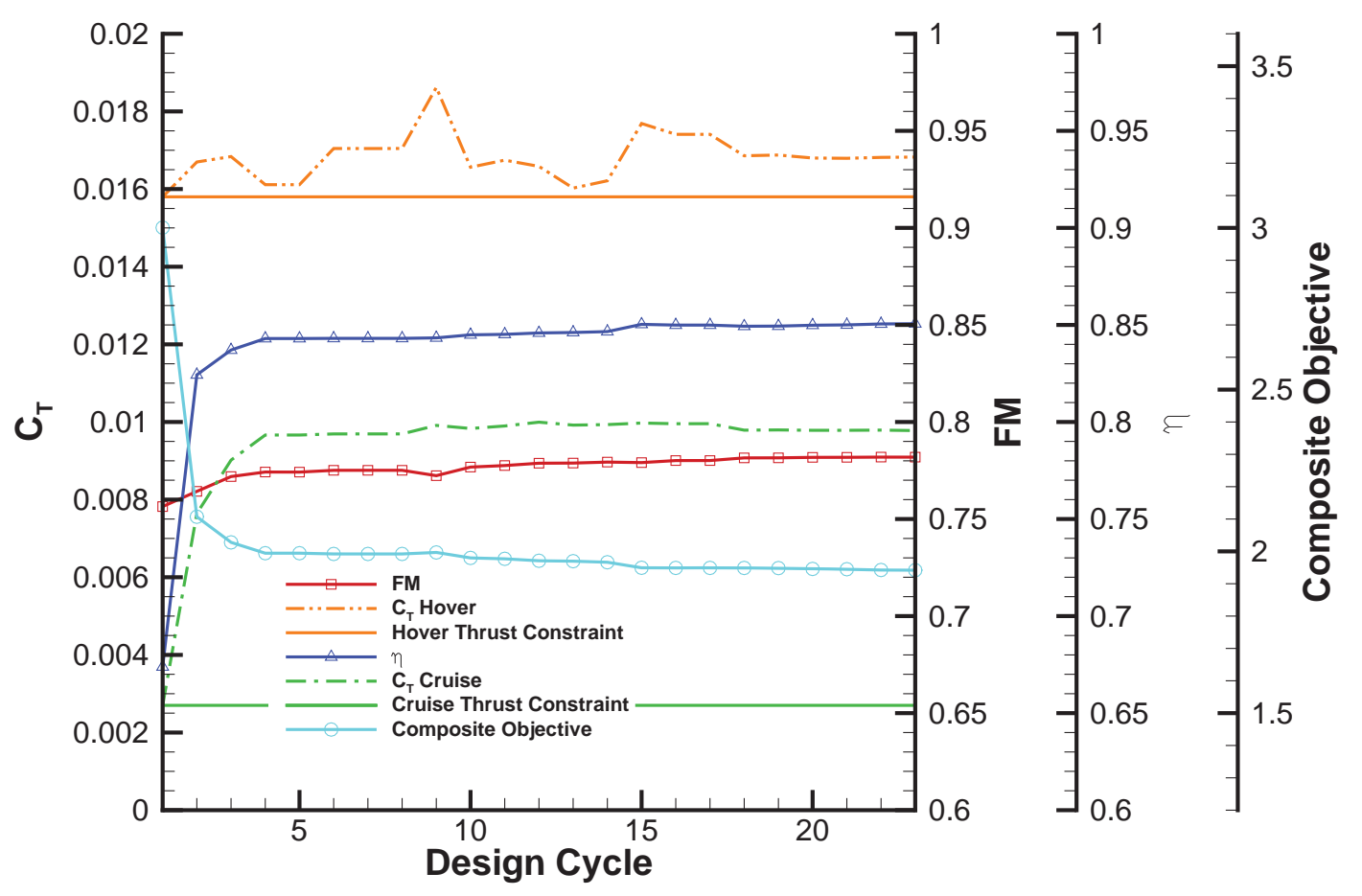

Figure 10. Step D: Composite Weight 1:2, Optimize Thickness, Camber, Twist, Taper, Collective. 


\section{References}

${ }^{1}$ Biedron, R. T. and Lee-Rausch, E. M., "Rotor Airloads Prediction Using Unstructured Meshes and Loose CFD/CSD Coupling," AIAA 2008-7341, Aug 2008.

${ }^{2}$ Boelens, O. J., van der Ven, H., Kok, J. C., and Prananta, B. B., "Rotorcraft Simulations Using a Sliding-Grid Approach," 34th European Rotorcraft Forum, Liverpool, U.K., 16-19 Sep 2008.

${ }^{3}$ Lee, H. K., Yoon, S. H., Shin, S. J., and Kim, C., "Coupled CFD/CSD Analysis of a Hovering Rotor Using High Fidelity Unsteady Aerodynamics and a Geometrically Exact Rotor Blade Analysis," 34th European Rotorcraft Forum, Liverpool, U.K., 16-19 Sep 2008.

${ }^{4}$ Lorber, P. F., Bagai, A., and Wake, B. E., "Design and Evaluation of Slatted Airfoils for Improved Rotor Performance," American Helicopter Society 62nd Annual Forum, 9-11 May 2006.

${ }^{5}$ Narramore, J. C., Lancaster, G., and Sheng, C., "Application of Computational Fluid Dynamics During the Conceptual Design of the Bell JHL Quad Tiltrotor," American Helicopter Society 63rd Annual Forum, 1-3 May 2007.

${ }^{6}$ O'Brien, D. M., Calvert, M. E., and Butler, S. L., "An Examination of Engine Effects on Helicopter Aeromechanics," American Helicopter Society Specialists Conference on Aeromechanics, 23-25 Jan 2008.

${ }^{7}$ Potsdam, M., Yeo, Y., and Johnson, W., "Rotor Airloads Prediction Using Loose Aerodynamic/Structural Coupling," Journal of Aircraft, Vol. 43, No. 3, 2006, pp. 732-742, doi: 10.2514/1.14006.

${ }^{8}$ Steijl, R. and Barakos, G., "Computational Analysis of Rotor-Fuselage Interactional Aerodynamics Using Sliding-Plane CFD Method," 34th European Rotorcraft Forum, Liverpool, U.K., 16-19 Sep 2008.

${ }^{9}$ Tanabe, Y. and Saito, S., "An Integrated Analysis Code with CFD/Rotor Dynamics Coupling Developed in JAXA," American Helicopter Society Specialists Conference on Aeromechanics, 23-25 Jan 2008.

${ }^{10}$ Modisette, J. M., An Output-Based Adaptive and Higher-Order Method for a Rotor in Hover, Master's thesis, Massachusetts Institute of Technology, Department of Aeronautics and Astronautics, Jan 2008.

${ }^{11}$ Nielsen, E. J. and Park, M. A., "Using an Adjoint Approach to Eliminate Mesh Sensitivities in Computational Design," AIA A Journal, Vol. 44, No. 5, May 2006, pp. 948-953.

${ }^{12}$ Park, M. A. and Darmofal, D. L., "Output-Adaptive Tetrahedral Cut-Cell Validation for Sonic Boom Prediction," AIAA 2008-6594, Aug 2008.

${ }^{13}$ Venditti, D. A., Grid Adaptation for Functional Outputs of Compressible Flow Simulations, Ph.D. thesis, Massachusetts Institute of Technology, Department of Aeronautics and Astronautics, 2002.

${ }^{14}$ Newman, III, J. C., Taylor, III, A. C., Barnwell, R. W., Newman, P. A., and Hou, G. J. W., "Overview of Sensitivity Analysis and Shape Optimization for Complex Aerodynamic Configurations," Journal of Aircraft, Vol. 36, No. 1, 1999, pp. 87-96, doi: $10.2514 / 2.2416$.

${ }^{15}$ Choi, S., Lee, K. H., Alonso, J. J., and Datta, A., "Preliminary Study on Time-Spectral and Adjoint-Based Design Optimization of Helicopter Rotors," American Helicopter Society Specialists Conference on Aeromechanics, 23-25 Jan 2008.

${ }^{16}$ Mavriplis, D. J., "Solution of the Unsteady Discrete Adjoint for Three-Dimensional Problems on Dynamically Deforming Unstructured Meshes," AIAA 2008-727, Jan 2008.

${ }^{17}$ Nielsen, E. J. and Diskin, B., "Discrete Adjoint-Based Design for Unsteady Turbulent Flows on Dynamic Overset Unstructured Grids," AIAA Journal, Vol. 51, No. 6, June 2013, pp. 1355-1373, doi: 10.2514/1.J051859.

${ }^{18}$ Nielsen, E. J., Lee-Rausch, E. M., and Jones, W. T., "Adjoint-Based Design of Rotors in a Noninertial Reference Frame," Journal of Aircraft, Vol. 47, No. 2, 2010, pp. 638-646, doi: 10.2514/1.46044.

${ }^{19}$ Anderson, W. K. and Bonhaus, D. L., "An Implicit Upwind Algorithm for Computing Turbulent Flows on Unstructured Grids," Computers and Fluids, Vol. 23, No. 1, 1994, pp. 1-21, doi: 10.1016/0045-7930(94)90023-X.

${ }^{20}$ Anderson, W. K., Rausch, R. D., and Bonhaus, D. L., "Implicit/Multigrid Algorithms for Incompressible Turbulent Flows on Unstructured Grids," Journal of Computational Physics, Vol. 128, No. 2, 1996, pp. 391-408, doi: 10.1006/jcph.1996.0219.

${ }^{21}$ Nielsen, E. J., Aerodynamic Design Sensitivities on an Unstructured Mesh Using the Navier-Stokes Equations and a Discrete Adjoint Formulation, Ph.D. thesis, Virginia Polytechnic Institute and State University, Department of Aerospace and Ocean Engineering, Dec 1998.

${ }^{22}$ Biedron, R. T. and Thomas, J. L., "Recent Enhancements to the FUN3D Flow Solver for Moving-Mesh Applications," AIAA 2009-1360, Jan 2009.

${ }^{23}$ Roe, P. L., "Approximate Riemann Solvers, Parameter Vectors, and Difference Schemes," Journal of Computational Physics, Vol. 43, No. 2, 1981, pp. 357-372, doi: 10.1016/0021-9991(81)90128-5.

${ }^{24}$ Spalart, P. R. and Allmaras, S. R., "A One-Equation Turbulence Model for Aerodynamic Flows," AIAA 92-0439, Jan 1991.

${ }^{25}$ Dacles-Mariani, J., Zilliac, G. G., Chow, J. S., and Bradshaw, P., "Numerical/Experimental Study of aWingtip Vortex in the Near Field," AIAA Journal, Vol. 33, No. 9, 1995, pp. 1561-1568, doi: 10.2514/3.12826.

${ }^{26}$ Saad, Y. and Schultz, M. H., "GMRES: A Generalized Minimal Residual Algorithm for Solving Nonsymmetric Linear Systems," SIAM Journal on Scientific and Statistical Computing, Vol. 7, No. 3, 1986, pp. 856-869, doi: 10.1137/0907058.

${ }^{27}$ Nielsen, E. J. and Anderson, W. K., "Recent Improvements in Aerodynamic Design Optimization on Unstructured Meshes," AIAA Journal, Vol. 40, No. 6, 2002, pp. 1155-1163, doi: 10.2514/2.1765.

${ }^{28}$ Nielsen, E. J., Lu, J., Park, M. A., and Darmofal, D. L., "An Implicit, Exact Dual Adjoint Solution Method for Turbulent Flows on Unstructured Grids," Computers and Fluids, Vol. 33, No. 9, 2004, pp. 1131-1155, doi: 10.1016/j.compfluid.2003.09.005.

${ }^{29}$ Samareh, J. A., "A Novel Shape Parameterization Approach," NASA TM-1999-209116, May 1999.

${ }^{30}$ Gill, P. E., Murray, W., and Saunders, M. A., "SNOPT: An SQP Algorithm For Large-Scale Constrained Optimization," SIAM Journal on Optimization, Vol. 12, 2002, pp. 979-1006.

${ }^{31}$ Gill, P. E., Murray, W., and Saunders, M. A., User's Guide for SNOPT Version 7: Software for Large-Scale Nonlinear Programming, Jun 162008.

${ }^{32}$ Young, L. A., Booth, Jr., E. R., Yamauchi, G. K., Botha, G., and Dawson, S., "Overview of the Testing of a Small-Scale Proprotor," American Helicopter Society 55th Annual Forum, Montreal, Canada, May 1999. 
${ }^{33}$ Swanson, S. M., McCluer, M. S., Yamauchi, G. K., and Swanson, A. A., "Airloads Measurements from a 1/4-Scale Tiltrotor Wind Tunnel Test," 25th European Rotorcraft Forum, Rome, Italy, Sep 1999.

${ }^{34}$ Pirzadeh, S., "Three-Dimensional Unstructured Viscous Grids by the Advancing Front Method," AIAA Journal, Vol. 34, No. 1, 1996, pp. 43-49, doi: 10.2514/3.13019.

${ }^{35}$ Kim, K. C., "Analytical Calculations of Helicopter Torque Coefficient $\left(C_{Q}\right)$ and Thrust Coefficient $\left(C_{T}\right)$ Values for the Helicopter Performance (HELPE) Model," ARL ARL-TR-1986, June 1999.

${ }^{36}$ Leishman, J., Principles of Helicopter Aerodynamics, Cambridge Aerospace Series, Cambridge University Press, 2002. 